\title{
Theoretical Study of the NMR Chemical Shift of Xe in Supercritical Condition
}

Lacerda Junior, Evanildo Gomes; Sauer, Stephan P. A.; Mikkelsen, Kurt Valentin; Coutinho, Kaline; Canuto, Sylvio

Published in:

Journal of Molecular Modeling

DOI:

10.1007/s00894-018-3600-4

Publication date:

2018

Document version

Peer reviewed version

Citation for published version (APA):

Lacerda Junior, E. G., Sauer, S. P. A., Mikkelsen, K. V., Coutinho, K., \& Canuto, S. (2018). Theoretical Study of the NMR Chemical Shift of Xe in Supercritical Condition. Journal of Molecular Modeling, 24, [62].

https://doi.org/10.1007/s00894-018-3600-4 


\section{Journal of Molecular Modeling Theoretical Study of the NMR Chemical Shift of Xe in Supercritical Condition --Manuscript Draft--}

Manuscript Number:

Full Title:

Article Type:

Keywords:

Corresponding Author:
JMMO-D-17-00857R1

Theoretical Study of the NMR Chemical Shift of Xe in Supercritical Condition

TC-SBQT2017

Xenon; Supercritical Condition; NMR chemical shift

Evanildo G. Lacerda Jr., Ph.D.

Universidade de Sao Paulo

BRAZIL

Corresponding Author Secondary Information:

Corresponding Author's Institution:

Universidade de Sao Paulo

Corresponding Author's Secondary Institution:

First Author:

Evanildo G. Lacerda Jr., Ph.D.

First Author Secondary Information:

Order of Authors:

Evanildo G. Lacerda Jr., Ph.D.

Stephan P. A. Sauer

Kurt V. Mikkelsen

Kaline Coutinho

Sylvio Canuto

Order of Authors Secondary Information:

Abstract:

Response to Reviewers:
In this work we investigate the level of theory necessary for reproducing the non-linear variation of the $\$ \wedge\{129\} \$$ Xe nuclear magnetic resonance (NMR) chemical shift with the density of Xe under supercritical conditions. In detail we study how the $\$^{\wedge}\{129\} \$ X e$ chemical shift depends under these conditions on electron correlation, relativistic and many-body effects. The latter are included using a sequential-QM/MM methodology, in which a classical MD simulation is performed first and the chemical shift is then obtained as an average of quantum calculations carried out for Xe $\$ n \$$ clusters ( $n=2-8$ depending on the density), whose geometries are taken from $250 \mathrm{MD}$ snapshots. The analysis of the relativistic effects is made at the level of 4-component Hartree-Fock calculations (4c-HF) and electron correlation effects are considered using second order M\{lo\}ller-Plesset perturbation theory (MP2). To simplify the calculations of the relativistic and electron correlation effects we adopted an additive scheme, where the calculations on the Xe\$_n\$ clusters are carried out at the non-relativistic Hartree-Fock (HF) level, while electron correlation and relativistic corrections are added for all the pairs of Xe atoms in the clusters. Using this approach we obtain very good agreement with the experimental data, showing that the chemical shift of $\$^{\wedge}\{129\} \$ X e$ in supercritical conditions is very well described by cluster calculations at the HF level, with small contributions from relativistic and electron correlation effects.

Detailed response to the reviewer 1's comments:

1) Reviewer's comment: "The temperature which MD simulation was carried on must be specified in the section 2.1."

Author's reply: Done.

2) Reviewer's comment: "In the section 2.2 it was not clear the comment about b3lyp data. Indeed these data was not reported and are not discussed throughout the text. Therefore, if the authors want to comment on b3lyp performance for NMR chemical 
shifts prediction the data and analysis must be clearly provided."

Author's reply: We have added our B3LYP results as SI and stated that the failure of B3LYP for Xe and noble gas dimers in general is a special case.

3) Reviewer's comment: "Figure 1 was obtained from 250 snapshots of the MD simulation at density of $1.151 \mathrm{~g} / \mathrm{cm} 3($ p. 3$)$. The point here is why not to construct the figure 1 using distinct interatomic distances for $\mathrm{Xe} 2$ ? What is the real difference between both approaches?"

Author's reply: The reason for using the distances obtained in the MD simulation is that they reflect which distances are really relevant for this system. This is mentioned in the text now.

4) Reviewer's comment: "Even though Eq. 2 was referenced to ref. 9, it would help the readers if a physical meaning for the correlation in Eq. 2 could be provided in the text." Author's reply: This is basically just a convenient fitting function which closely fits the data, although it probably was motivated by the fact that the interaction between noble gases is a dispersion interaction.

5) Reviewer's comment: "Figure 6 was not clear and I didn't find it essential to understand the analysis of the clusters' size."

Author's reply: We would like to keep this figure and have therefore extended the description in the text.

6) Reviewer's comment: "The calculation of the MP2 and 4c-HF average corrections provided in Table 3 could be explained in the main text or as a footnote."

Author's reply: Done.

Detailed response to the reviewer 2's comments:

1) Reviewer's comment: "Pg. 2. The number of 250 snapshots from a MD simulation could be justified."

Author's reply: Done see comment 3 above.

2) Reviewer's comment: "The following references could be introduced in the paper: Magnetic Resonance in Chemistry. Vol.77, 1399-1408 (2017); J Comput Chem. Vol. 15; 1890-8 (2013)"

Author's reply: Yes, of course.

Yours sincerely

Evanildo G. Lacerda Jr. 


\title{
Theoretical Study of the NMR Chemical Shift of Xe in Supercritical Condition
}

\author{
Evanildo G. Lacerda Jr. • Stephan P. A. Sauer • Kurt V. Mikkelsen • \\ Kaline Coutinho • Sylvio Canuto
}

Received: date / Accepted: date

\begin{abstract}
In this work we investigate the level of theory necessary for reproducing the non-linear variation of the ${ }^{129}$ Xe nuclear magnetic resonance (NMR) chemical shift with the density of Xe in supercritical conditions. In detail we study how the ${ }^{129} \mathrm{Xe}$ chemical shift depends under these conditions on electron correlation, relativistic and many-body effects. The latter are included using a sequential-QM/MM methodology, in which a classical MD simulation is performed first and the chemical shift is then obtained as an average of quantum calculations of $250 \mathrm{MD}$ snapshots conformations carried out for $\mathrm{Xe}_{n}$ clusters ( $n=2-8$ depending on the density). The analysis of the relativistic effects is made at the level of 4-component Hartree-Fock calculations (4c-HF) and electron correlation effects are considered using second order Møller-Plesset perturbation theory (MP2). To simplify the calculations of the relativistic and electron correlation effects we adopted an additive scheme, where the calculations on the $\mathrm{Xe}_{n}$ clusters are carried out at the non-relativistic Hartree-Fock (HF) level, while electron correlation and relativistic corrections are added for all the pairs of Xe atoms in the clusters. Using this approach we obtain very good
\end{abstract}

Evanildo G. Lacerda Jr.

Department of Chemistry, University of Copenhagen, Universitetsparken 5, DK-2100 Copenhagen $\varnothing$, Denmark

E-mail: evanildo@if.usp.br Present address: Instituto de Física da Universidade de São Paulo, Rua do Matão 1371, 05508-090 São Paulo, SP, Brazil

Stephan P. A. Sauer, Kurt V. Mikkelsen

Department of Chemistry, University of Copenhagen,

Universitetsparken 5, DK-2100 Copenhagen $\varnothing$, Denmark

E-mail: sauer@kiku.dk, kmi@chem.ku.dk

Kaline Coutinho, Sylvio Canuto

Instituto de Física da Universidade de São Paulo,

Rua do Matão 1371, 05508-090 São Paulo, SP, Brazil

E-mail: kaline@if.usp.br, canuto@if.usp.br agreement with the experimental data, showing that the chemical shift of ${ }^{129} \mathrm{Xe}$ in supercritical conditions is very well described by cluster calculations at the HF level, with small contributions from relativistic and electron correlation effects.

Keywords Xenon · Supercritical Condition · NMR chemical shift

\section{Introduction}

Xenon in supercritical condition can be used as a powerful tool to investigate various chemical systems. On one hand supercritical fluids are particularly interesting since slight chances in thermodynamic condition modifies the dielectric properties allowing to tune the solvent effects[1]. They have therefore found many applications as solvent for organic reactions, separations processes, and hazardous waste destruction [2]. On the other hand, Xenon is lipophilic and soluble in many substances [3]. With its highly sensitive NMR chemical shift Xenon serves as a probe used to characterize solids [4], proteins and biomembranes [5,6], blood [7], nanosystems [8], polymers, clathrates, mixtures [9], and even to detect radiations of interest in astrophysics [10]. Consequently, gaseous, liquid and supercritical Xenon has been the subject of experimental investigation as well as theoretical calculations $[11,12]$.

In this work we explore how the NMR chemical shift of ${ }^{129} \mathrm{Xe}$ depends on the internal atomic structure in the supercritical condition, many-body effects, relativistic effects obtained from 4-component HartreeFock calculations (4c-HF) and electron correlation effects through second order Møller-Plesset perturbation theory (MP2). Furthermore, for proper average over many configurations of Xe clusters with with a large 
number of electrons it is important to find a way to reduce the computational time in calculating electron correlation and relativistic effects. The idea consists in decomposing the chemical shift in various contributions, where the Xe system in the specified thermodynamic condition is generated by Molecular Dynamics (MD) simulations and by averaging of chemical shifts calculated at the non-relativistic Hartree-Fock level for $\mathrm{Xe}_{n}$ clusters with configurations taken from 250 snapshots of the MD simulations. The number $n$ of Xe atoms in the clusters will hereby depend on the chosen density. The electron correlation and relativistic effects will afterwards be treated at the two-body level, i.e. correlation and relativistic corrections will be added for each pair of Xe atoms in a given clusters.

\section{Computational Details}

\subsection{Molecular Dynamics Simulations}

The molecular dynamics simulations were carried out with the Gromacs package $[13,14]$, where the Xenon atoms were described by parameters suggested by Verlet and Weis [15] for the van der Waals interactions. We performed the MD simulations in the NVT ensemble for the densities of $0.2878,0.8633,1.151$ and 1.727 $\mathrm{g} / \mathrm{cm}^{3}$ and temperature of $298 \mathrm{~K}$. A total of $1000 \mathrm{Xe}$ atoms were included in the simulation cubic box. The equations of motion were integrated using the leap-frog algorithm, with a time step of $1 \mathrm{fs}$. The system was thermalized for $1 \mathrm{~ns}$ and the total equilibrium simulation time was $5 \mathrm{~ns}$. The neighbor list was updated at each 10 fs. All energy terms were computed until the cut-off ratio of $10 \AA$, and long-range corrections for the Lennard-Jones interactions were calculated for distances beyond the cut-off ratio using the analytical integral of an uniform density. The Berendsen barostat and thermostat $[16,17]$ were employed, with coupling constants of $0.1 \mathrm{ps}$ for the temperature, and $1.0 \mathrm{ps}$ for the pressure. For each 20 ps a snapshot was saved for the quantum calculations leading to 250 snapshots in total in order to ensure the convergence of the average calculated chemical shift. For the radial distribution function 50000 snapshots were used.

\subsection{Quantum Mechanical Calculations}

The non-relativistic quantum calculations of the shielding constant at the Hartree-Fock level and the level of second order Møller-Plesset perturbation theory were carried out with the Gaussian 09 program [18]. In these calculations the DZP [19], TZP [20] and AQZP [21] basis sets of Jorge and co-workers were used, which were downloaded from the Environmental Molecular Sciences Laboratory (EMSL) basis set library [22,23]. The relativistic 4c-HF calculations were carried out with the DIRAC 14 program [24-27] using the av4z basis set of Dyall [28]. In preliminary density functional theory calculations with the B3LYP exchange-correlation functional we observed that they predict a significant amount of electron correlation contribution to the chemical shift of Xe dimers (see SI), which is neither confirmed by our present MP2 results nor by previous MP2 and $\operatorname{CCSD}(\mathrm{T})$ results [12]. The failure of B3LYP for Xe dimers is special for noble gas dimers and no general conclusion should be drawn from that. These DFT/B3LYP results will therefore not be discussed further in the following.

The chemical shift, $\delta$, for Xenon is defined approximately as

$\delta \cong \sigma_{\text {isolated }}-\sigma_{\text {env }}$,

where the reference value $\sigma_{\text {isolated }}$ is the absolute nuclear magnetic shielding constant for the isolated atom and $\sigma_{\text {env }}$ is the shielding constant for the atom surrounded by some environment. In theoretical calculations of the chemical shift both terms should be obtained at the same level of theory.

\section{Results}

\subsection{Calculations for the Isolated Xenon}

As a starting point, we calculated the absolute shielding constant for the isolated Xenon atom using the AQZP basis set in the non-relativistic calculations and the av $4 \mathrm{z}$ in the relativistic calculations. The results are $5644.85 \mathrm{ppm}$ at the non-relativistic HF level, 5644.45 ppm at the non-relativistic MP2 level and $7020.76 \mathrm{ppm}$ at the $4 \mathrm{c}-\mathrm{HF}$ level. The non-relativistic calculations yield virtually identical results, whereas we obtain a large relativistic correction to the absolute shielding constant in very good agreement with previous calculations [12]. These values will be used in the following as reference values for the calculation of the chemical shifts according to Eq. (1).

\subsection{Mean Field, Electron Correlation and Relativistic} Effects for the Xe Dimer

For the final calculations of the chemical shift of Xe at different densities, we calculate the chemical shift for $\mathrm{Xe}_{n}$ clusters with varying interatomic distances. In this 


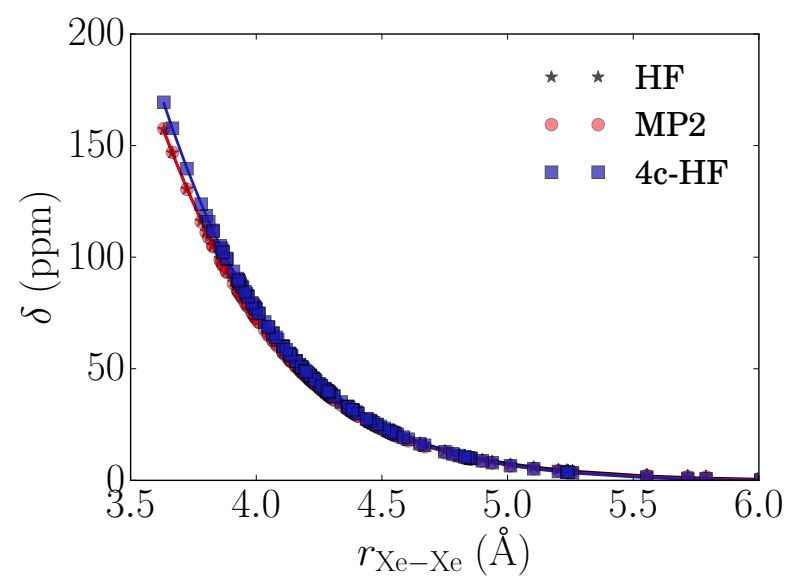

Fig. 1 Calculated chemical shifts of a Xe dimer in ppm as a function of the interatomic distance.

section we will therefore study at different levels of theory, how the chemical shift changes, when we vary the distance between two Xe atoms. Since larger electron correlation effects are expected for closer interatomic distances due to the increasing overlap between the electron densities, we were in particular interested in obtaining how HF and MP2 or the relativistic 4c-HF results for the changes are compared with each other. The AQZP basis set was used for the non-relativistic calculations and the av $4 \mathrm{z}$ basis set for the relativistic ones. In order to sample interatomic distances more likely to occur, we selected pairs from the 250 snapshots of the MD simulation at the supercritical density of 1.151 $\mathrm{g} / \mathrm{cm}^{3}$. Plotting the results in Figure 1 we see that for distances greater than approximately $6.0 \AA$ the values of the chemical shift $\delta$ are equal to zero implying that at these distances there is no effect anymore of one Xe atom on the shielding of the other. The similarity between HF and MP2 chemical shift curves indicates that electron correlation effects do not play a major role in the calculated shifts for pairs of Xe atoms in agreement with the earlier $\operatorname{CCSD}(\mathrm{T})$ results for the equilibrium dimer geometry [12].

From the calculated values we can adjust an empirical function [11] to obtain the expected values of the chemical shift $\delta_{\text {pair }}$ given the distance $r$ between pairs of Xe atoms, using

$\delta_{\text {pair }}=\frac{A}{r^{6}}+\frac{B}{r^{8}}+\frac{C}{r^{10}}+\frac{D}{r^{12}}+\frac{E}{r^{14}}$,

where the adjusted parameters, obtained by a leastsquare fit, are given in Table 1.

By adding the contribution of each surrounding Xe atom in a Xe cluster using this function we can calculate the two-body approximation to the chemical shift of the central Xe atom in the cluster and thus the average 2-
Table 1 Adjustable parameters in the empirical function Eq. (2) for the chemical shift, $\delta_{\text {pair }}$, in Xe dimers.

\begin{tabular}{cccc}
\hline & HF & MP2 & $4 \mathrm{c}-\mathrm{HF}$ \\
\cline { 2 - 4 }$A$ & $-3.434591 \times 10^{4}$ & $-6.587426 \times 10^{4}$ & $-1.297478 \times 10^{5}$ \\
$B$ & $-4.309107 \times 10^{6}$ & $-3.765209 \times 10^{6}$ & $-1.145628 \times 10^{6}$ \\
$C$ & $2.918698 \times 10^{8}$ & $2.903287 \times 10^{8}$ & $2.562887 \times 10^{8}$ \\
$D$ & $-2.197023 \times 10^{9}$ & $-2.196882 \times 10^{9}$ & $-1.992953 \times 10^{9}$ \\
$E$ & 1.000024 & 1.000007 & 1.000970 \\
\hline
\end{tabular}

body chemical shift $\left\langle\delta_{2 \mathrm{~b}}\right\rangle$ for a set of clusters without carrying out further quantum calculations.

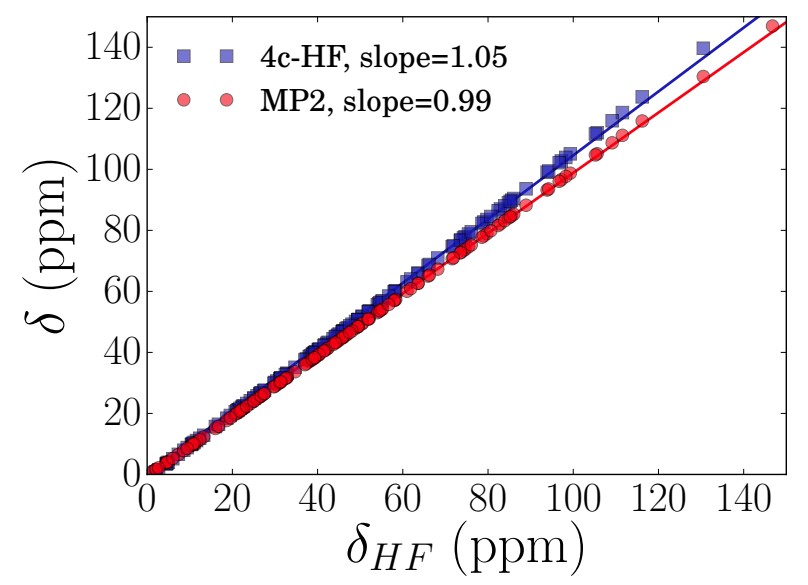

Fig. 2 The correlation of the chemical shifts in Xe dimers calculated at MP2 and 4c-HF levels with the corresponding $\mathrm{HF}$ values in ppm.

However, plotting the chemical shifts at the MP2 and $4 \mathrm{c}-\mathrm{HF}$ levels against the corresponding $\mathrm{HF}$ values (see Figure 2), we noticed that the points fit a linear function $\delta \approx a \delta_{\mathrm{HF}}$, which allows us to obtain a scale factor relating these methods. The slopes $a$ are 0.99 for MP2 and 1.05 for 4c-HF. Expressed in other words, the chemical shifts of Xe dimers compared to the HF level are $5 \%$ higher at the 4c-HF level and $1 \%$ lower at the MP2 level.

\subsection{Coordination Shell}

From the MD simulation we can characterize the coordination shell of Xe at the different densities using the radial distribution function (RDF) and the average number of surrounding Xe atoms. In Figure 3 we show the radial distribution function (RDF) for different densities. As one would expect, the first peak becomes sharper as the density increases, indicating closer interactions. The integration of the RDF until $6.0 \AA$ gives 


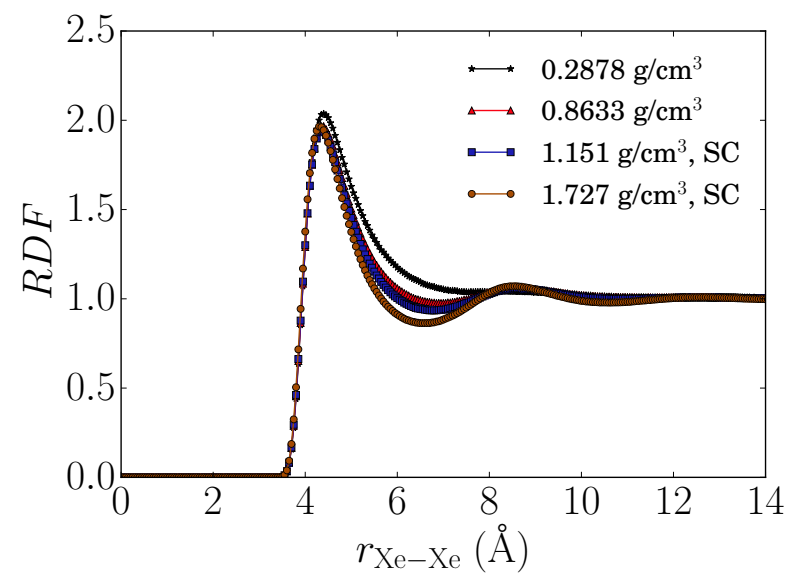

Fig. 3 Radial distribution function (RDF) for Xenon in different densities calculated from 50000 snapshots of the MD simulations. SC indicates densities larger than the critical density.

a coordination number of $1.3 \mathrm{Xe}$ atoms for the density $0.2878 \mathrm{~g} / \mathrm{cm}^{3}, 3.8$ for $0.8633 \mathrm{~g} / \mathrm{cm}^{3}, 4.8$ for $1.151 \mathrm{~g} / \mathrm{cm}^{3}$ and 7.0 for $1.727 \mathrm{~g} / \mathrm{cm}^{3}$.

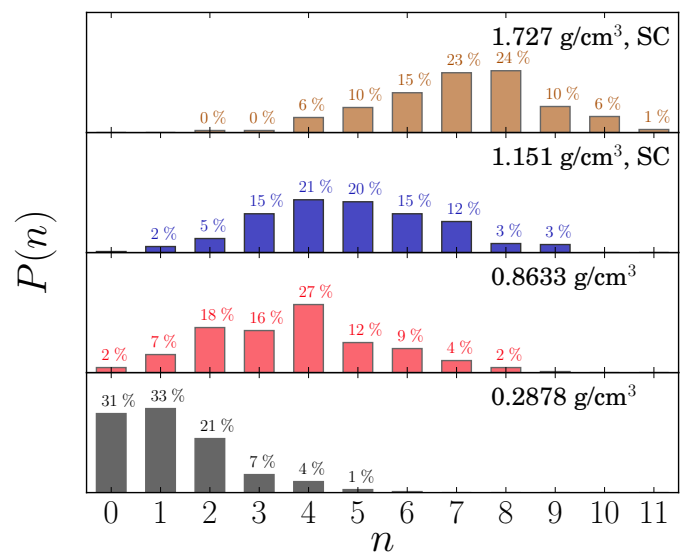

Fig. 4 Distribuition of $n$ neighbor atoms in the coordination shell $(r<6.0 \AA)$. For the 250 configurations selected to quantum calculations.

Since atoms separated with distances higher than $6.0 \AA$ have no effect on the chemical shift of Xenon (see Figure 1), we define the first coordination shell as the sphere centered on the central Xe atom having a radius of $6.0 \AA$. The average number of surrounding atoms inside this sphere defines an effective coordination number $n$. We calculated the distribution of $n$ in the samples of 250 snapshots taken from the different MD simulations. The distributions, in Figure 4, show that for $0.2878 \mathrm{~g} / \mathrm{cm}^{3}$ around $31 \%$ of the configurations have no other atom in the coordination shell. On the other hand, for $1.151 \mathrm{~g} / \mathrm{cm}^{3}$ the $n$ values have a broader distribution. We note further, from Figure 5, that there is a linear correlation between the average effective coordination number $\langle n\rangle$ and the density of the Xe fluid.

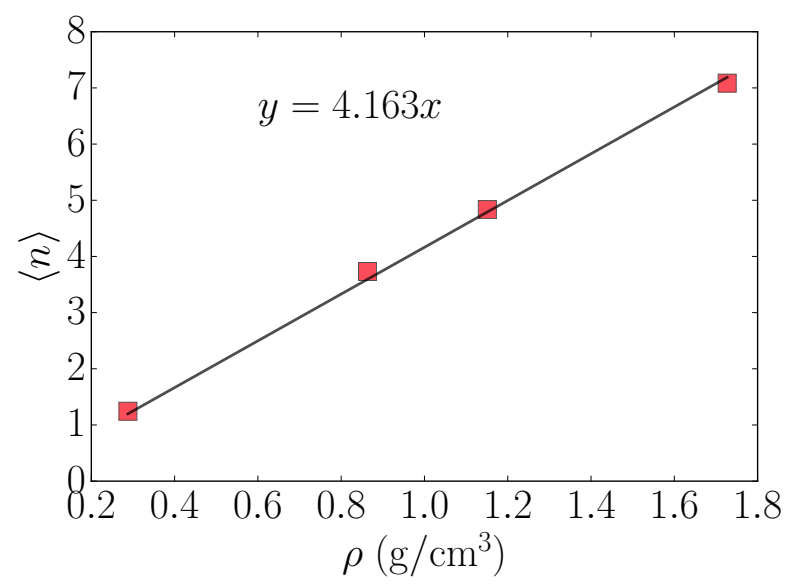

Fig. 5 Relationship between the average effective coordination number $\langle n\rangle$ and the density $\rho$ of the Xe fluid.

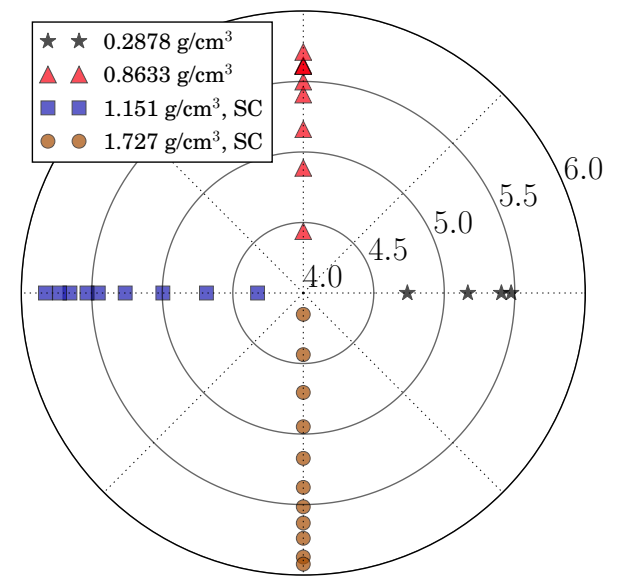

Fig. 6 Average distance (in $\AA$ ) to the neighbor atoms in the first shell.

Since the interatomic distances play a central role for the chemical shifts of Xe dimers, we have also analyzed how close the next Xe atoms get depending on the density. In Figure 6 we plotted thus the average distance to the closest neighboring atom, the second closest neighboring atom and so on with the results for the four different densities separated on the axis and the distances as circles from the origin starting with a distance of $4.0 \AA$. As a general trend one clearly sees that 
on increasing density the distance to the closest atom decreases and the number of closer $(r<6 \AA)$ atoms increases. The distances to the closest neighbor atom are $4.7 \AA\left(0.2878 \mathrm{~g} / \mathrm{cm}^{3}\right), 4.4 \AA\left(0.8633 \mathrm{~g} / \mathrm{cm}^{3}\right), 4.3 \AA(1.151$ $\left.\mathrm{g} / \mathrm{cm}^{3}\right)$ and $4.2 \AA\left(1.727 \mathrm{~g} / \mathrm{cm}^{3}\right)$. Although these are not very different values, it is worth to remember that the Xe chemical shift in the dimers responds non-linearly to the distances, see Figure 1.

\subsection{Basis Set Dependence of Chemical Shift in Xe Clusters}

We investigate how the basis set on the surrounding Xe atoms affects the calculated average chemical shift of the central Xe atom, $\left\langle\delta_{\mathrm{HF}}\right\rangle$, in $250 \mathrm{MD}$ configuraions of clusters within a radius of $6.0 \AA$. The results obtained at the HF level in Table 2 show that one can obtain different values for $\left\langle\delta_{\mathrm{HF}}\right\rangle$ if the basis set on the surrounding Xe atoms is DZP, TZP or AQZP, resulting in $67.4 \mathrm{ppm}, 69.8 \mathrm{ppm}$ and $82.1 \mathrm{ppm}$ respectively, a spread of almost $15 \mathrm{ppm}$ from DZP to AQZP. However, using the AQZP basis set for the closest one or two Xe atoms, denoted as "1 AQZP + DZP" and "2 AQZP + DZP" in Table 2, we could obtain values in better agreement with the results with the AQZP basis set on all surrounding Xe atoms, i.e. $75.9 \mathrm{ppm}$ or 80.3 ppm. The latter is close to the value of $82.1 \mathrm{ppm}$ for the full AQZP description of the cluster. It is possible to understand why the improvement in the basis set for the closest two molecules is enough for obtaining converged results, if we look again at the average distance of each neighbor atom in Figure 6. For the density of $1.151 \mathrm{~g} / \mathrm{cm}^{3}$ the two closest surrounding atoms are positioned at 4.3 and $4.7 \AA$, and the third closest atom at $5.0 \AA$. As one can see in Figure 1, for distance larger than $5.0 \AA$ the contribution to the chemical shift is very small, which explains why we only need to improve the basis set for the first and second closest atoms.

\subsection{Many-body Effects on the Chemical Shift in Xe} Clusters

The results of the chemical shifts averaged over the 250 snapshots and using different models for the many-body effects are compared in Table 3 with the experimental values at the different densities. In the 2-body approximation, $\left\langle\delta_{\mathrm{HF}-2 \mathrm{~b}}\right\rangle$, the chemical shift of $\mathrm{Xe}$ in a given cluster is simply the sum of contributions, $\delta_{\text {pair }}$, from each pair of the central Xe atom with one of its surrounding Xe atoms. The value of these pair contributions at the HF level was then obtained from Eq.(2) and Table 1 . From Table 3 one can see that this model
Table 2 Dependence of the averaged Xe chemical shift (in ppm) at the HF level, $\left\langle\delta_{\mathrm{HF}}\right\rangle$, on the basis set on the surrounding Xe atoms for a density of $1.151 \mathrm{~g} / \mathrm{cm}^{3}$. The average number of Xe atoms in the cluster is 5 and the average was performed over $250 \mathrm{MD}$ configurations.

\begin{tabular}{lc}
\hline Basis set & $\left\langle\delta_{\mathrm{HF}}\right\rangle$ \\
\hline DZP & 67.4 \\
TZP & 69.8 \\
1 AQZP + DZP & 75.9 \\
2 AQZP + DZP & $b$ \\
AQZP & 80.3 \\
\hline EXP[3] & 82.4 \\
\hline${ }^{a}$ AQZP basis set for the closest Xe atom, DZP basis set for \\
the others. \\
bQZ basis set for the two closest Xe atoms, DZP basis \\
set for the others.
\end{tabular}

overestimates in general the chemical shift for higher densities leading to absolute errors of $1.4 \mathrm{ppm}(0.2878$ $\left.\mathrm{g} / \mathrm{cm}^{3}\right), 8.4 \mathrm{ppm}\left(0.8633 \mathrm{~g} / \mathrm{cm}^{3}\right), 9.4 \mathrm{ppm}\left(1.151 \mathrm{~g} / \mathrm{cm}^{3}\right)$ and $23.3 \mathrm{ppm}\left(1.727 \mathrm{~g} / \mathrm{cm}^{3}\right)$. The good agreement for the lower gas-like density of $0.2878 \mathrm{~g} / \mathrm{cm}^{3}$ can be explained by the effective coordination number $\langle n\rangle$, Figure 5 , which equals only 1.3 , implying that on average there is not much more than one neighboring Xe atom at this density.

Table 3 Averaged Xe chemical shifts (in ppm) calculated with different models of treating many-body effects.

\begin{tabular}{lrrrr}
\hline & \multicolumn{4}{c}{ Density in $\mathrm{g} / \mathrm{cm}^{3}$} \\
\cline { 2 - 5 }$\left\langle\delta_{\mathrm{HF}-2 \mathrm{~b}}\right\rangle$ & 0.2878 & 0.8633 & 1.151 & 1.727 \\
\cline { 2 - 5 }$\left\langle\Delta_{\mathrm{MP}-2 \mathrm{~b}}\right\rangle$ & 24.2 & 73.9 & 89.8 & 141.2 \\
$\left\langle\Delta_{4 \mathrm{c}-\mathrm{HF}-2 \mathrm{~b}}\right\rangle$ & -0.9 & -3.0 & -3.6 & -5.4 \\
& 0.0 & -0.3 & -0.4 & 0.0 \\
$\left\langle\delta_{\mathrm{HF}-1 \mathrm{st}}\right\rangle$ & 22.8 & 64.8 & 82.1 & 121.7 \\
$\left\langle\delta_{\mathrm{HF}}\right\rangle$ & 24.2 & 65.4 & 82.4 & 122.1 \\
$\left\langle\delta_{\mathrm{HF}}\right\rangle+\langle\Delta\rangle^{\mathrm{a}}$ & 23.3 & 62.1 & 78.4 & 116.7 \\
$\mathrm{EXP}[3]$ & 25.6 & 65.5 & 80.4 & 117.9 \\
\hline $\mathrm{a}\langle\Delta\rangle=\left\langle\Delta_{\mathrm{MP} 2-2 \mathrm{~b}}\right\rangle+\left\langle\Delta_{4 \mathrm{c}-\mathrm{HF}-2 \mathrm{~b}}\right\rangle$ & &
\end{tabular}

In Table 3 we show how much the chemical shifts evaluated with the 2-body approximation would change, if the pair contributions, $\delta_{\text {pair }}$, would have been calculated at the MP2 level, $\left\langle\Delta_{\mathrm{MP} 2-2 \mathrm{~b}}\right\rangle$, or at the $4 \mathrm{c}-\mathrm{HF}$ level, $\left\langle\Delta_{4 \mathrm{c}-\mathrm{HF}-2 \mathrm{~b}}\right\rangle$. These corrections were obtained by computing the difference between the MP2 or $4 \mathrm{c}-\mathrm{HF}$ value and the corresponding $\mathrm{HF}$ value for each solutesolvent pair in a snapshot from Eq.(2) and Table 1 and afterwards averaging over the 250 snapshots. The electron correlation corrections are negative and in the order of $4 \%$ in agreement with Figure 2. The relativistic corrections are even smaller, but are negative and have a maximum for a density of $0.8633 \mathrm{~g} / \mathrm{cm}^{3}$. This can be understood from Figure 7 showing that the rel- 
ativistic corrections to the chemical shift in the Xe dimers are positive for interatomic distances smaller than $\sim 4.5 \AA$, but become negative for larger distances. However, there are no neighboring atoms closer than $4 \AA$, Figure 6 , where there would be a large positive relativistic correction, and the total contribution of the neighbor atoms depends than critically on their distribution below and above $\sim 4.5 \AA$.

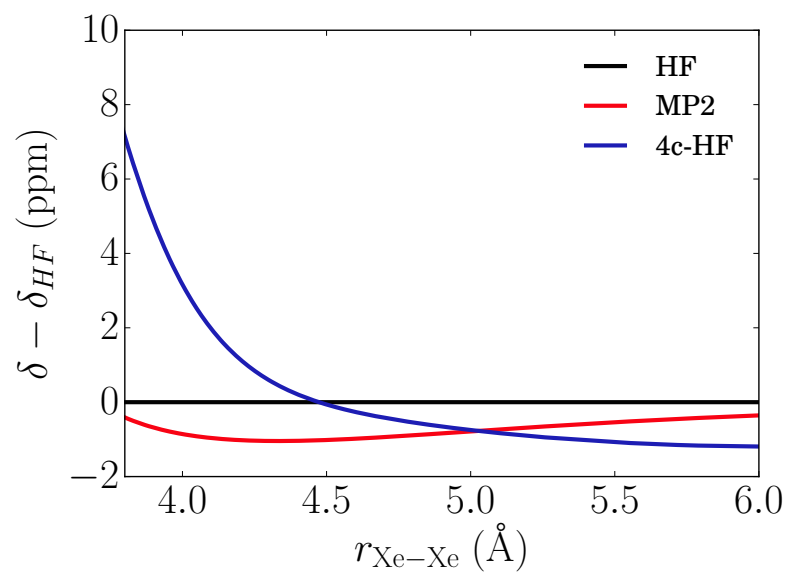

Fig. 7 Dependence of the electron correlation correction at the MP2 level and the relativistic correction at the $4 \mathrm{c}-\mathrm{HF}$ level to the chemical shift (in ppm) of the Xe dimer on the interatomic distance.

Considering 3-body and higher order effects by carrying out $\mathrm{HF} / \mathrm{AQZP}$ calculations on Xe clusters for each snapshot, $\left\langle\delta_{\mathrm{HF}-1 \mathrm{st}}\right\rangle$ and $\left\langle\delta_{\mathrm{HF}}\right\rangle$, we substantially improve the results. They were calculated using two different ways for selecting the number of atoms in the clusters. In the first approach, $\left\langle\delta_{\mathrm{HF}-1 \mathrm{st}}\right\rangle$, which is frequently employed in the literature, the clusters contained in each snapshot the same number of atoms, i.e. $\mathrm{Xe}_{\langle n\rangle+1}$, while in the second approach, $\left\langle\delta_{\mathrm{HF}}\right\rangle$, the clusters contained in all snapshots all the Xe atoms up to $6.0 \AA$ away from the central atom, a number which varies with the snapshot. The results with both approaches are for all the larger densities in excellent agreement with the experimental data. The absolute errors are for $\left\langle\delta_{\mathrm{HF}-1 \mathrm{st}}\right\rangle 0.7 \mathrm{ppm}\left(0.8633 \mathrm{~g} / \mathrm{cm}^{3}\right), 1.7$ $\left(1.151 \mathrm{~g} / \mathrm{cm}^{3}\right)$ and $3.8 \mathrm{ppm}\left(1.727 \mathrm{~g} / \mathrm{cm}^{3}\right)$ and for $\left\langle\delta_{\mathrm{HF}}\right\rangle$ $0.1 \mathrm{ppm}\left(0.8633 \mathrm{~g} / \mathrm{cm}^{3}\right), 2.0 \mathrm{ppm}\left(1.151 \mathrm{~g} / \mathrm{cm}^{3}\right)$ and $4.2 \mathrm{ppm}\left(1.727 \mathrm{~g} / \mathrm{cm}^{3}\right)$. Only for the smallest density, $0.2878 \mathrm{~g} / \mathrm{cm}^{3}$, the approach with a fixed cluster size shows a larger deviation of $2.8 \mathrm{ppm}$ compared to the approach with clusters containing all the atoms up to $6.0 \AA, 1.4 \mathrm{ppm}$. The explanation for this can be found in Figure 4, showing that only in $64 \%$ of the snapshots the coordination shell consists of one atom or less for
$0.2878 \mathrm{~g} / \mathrm{cm}^{3}$, while in $36 \%$ more atoms coordinate the central Xe atom. The contributions of these atoms to the chemical shift are, however, not included in this approximation. Adding the 2-body corrections for electron correction and relativity improves the agreement even further for the largest density.

In Figure 8 the dependence on the density of the calculated and experimental chemical shifts is shown. The dashed line refers to the extrapolation of the experimental low density chemical shifts. The 2-body approximation for the chemical shift at the HF level $\left\langle\delta_{\mathrm{HF}-2 \mathrm{~b}}\right\rangle$ overestimates the chemical shifts for higher densities, staying closer to the dashed line, whereas the full cluster calculations at the $\mathrm{HF}$ level, $\left\langle\delta_{\mathrm{HF}}\right\rangle$, or at the $\mathrm{HF}$ level with the 2-body electron correlation and relativity corrections, $\left\langle\delta_{\mathrm{HF}}\right\rangle+\left\langle\Delta_{\mathrm{MP} 2-2 \mathrm{~b}}\right\rangle+\left\langle\Delta_{4 \mathrm{c}-\mathrm{HF}-2 \mathrm{~b}}\right\rangle$, perfectly reproduces the experimental density dependence.

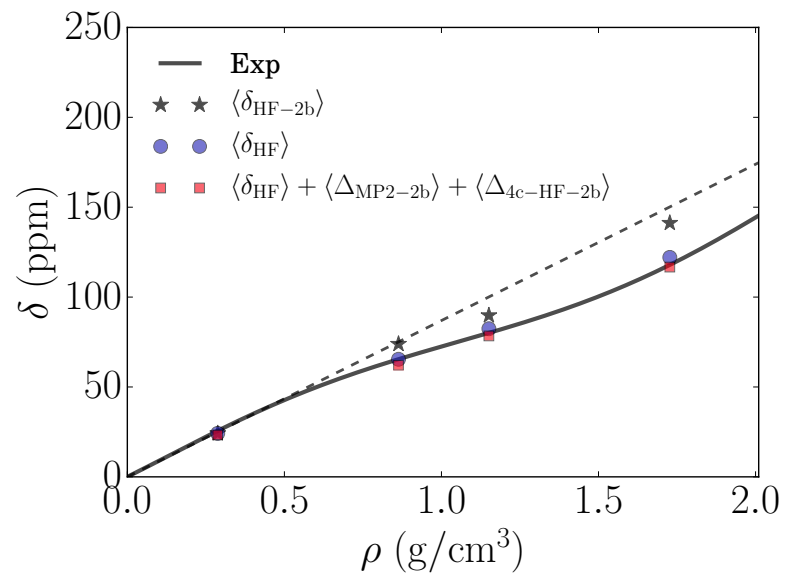

Fig. 8 Experimental [3] and calculated chemical shifts for $\mathrm{Xe}$ as a function of the density $\rho$. The dashed line refer to the low density extrapolation of the experimental chemical shift.

\section{Conclusions}

We have investigated electron correlation, basis set, relativistic and many-body effects that could influence the chemical shifts of Xe at densities below and above the critical density. Electron correlation and relativistic effects are found to play only a very minor role. But many-body effects among the Xe atoms are found to be necessary for explaining the non-linear behavior of the chemical shift as the density increases. Even at the non-relativistic HF level such many-body effects can be obtained sufficiently accurately for Xe, opening the door for studies with more complicated environments. 
Acknowledgements EGL thanks CNPq and the Science without Borders program for a postdoc fellowship (206886/20144), KC and SC thank CNPq for the grants (30437/2014-9 and 305203/2014-2, respectively), KVM, SPAS and KC thank CNPq for PVE project (401187/2014-4). SPAS and KVM thank the Danish Center for Scientific Computing for financial support.

\section{References}

1. Hrnjez BJ, Kabarriti A, Dach BI, Buldyrev SV, Asherie N, Natanov GR, Balderman J (2008) Pyrazine in supercritical Xenon: Local number density defined by experiment and calculation. J Phys Chem B 112:15431-15441. https://doi.org/10.1021/jp807586d

2. Pfund DM, Zemanian TS, Linehan JC, Fulton JL, Yonker CR (1994) Fluid Structure in Supercritical Xenon by Nuclear Magnetic Resonance Spectroscopy and Small Angle X-ray Scattering. J Phys Chem 98:11846-11857. https://doi.org/10.1021/j100097a009

3. Baumer D, Fink A, Brunner E (2003) Measurement of the ${ }^{129} \mathrm{Xe}$ NMR Chemical Shift of Supercritical Xenon. Zeitschrift für Phys Chemie 217:289-293. https://doi.org/10.1524/zpch.217.3.289.20465

4. Omi H, Nagasaka B, Miyakubo K, Ueda T, Eguchi $\mathrm{T}$ (2004) High-pressure ${ }^{129} \mathrm{Xe}$ NMR study of supercritical xenon confined in the mesopores of FSM-16. Phys Chem Chem Phys 6:1299-1303. https://doi.org/10.1039/b314841f

5. Miller KW, Reo NV, Schoot Uiterkamp AJ, Stengle DP, Stengle TR, Williamson KL (1981) Xenon NMR: chemical shifts of a general anesthetic in common solvents, proteins, and membranes. Proc Natl Acad Sci USA 78:4464949. https://doi.org/10.1073/pnas.78.8.4946

6. Yuan H, Murad S, Jameson CJ, Olson JD (2007) Molecular dynamics simulations of Xe chemical shifts and solubility in n-alkanes. J Phys Chem C 111:15771-15783. https://doi.org/10.1021/jp0735233

7. Norquay G, Leung G, Stewart NJ, Wolber J, Wild JM (2017) ${ }^{129}$ Xe chemical shift in human blood and pulmonary blood oxygenation measurement in humans using hyperpolarized ${ }^{129}$ Xe NMR. Magn Reson Med 77:13991408. https://doi.org/10.1002/mrm. 26225

8. Standara S, Kulhánek P, Marek R, Straka M (2013) ${ }^{129} \mathrm{Xe}$ NMR chemical shift in $\mathrm{Xe} @ \mathrm{C}_{60}$ calculated at experimental conditions: Essential role of the relativity, dynamics, and explicit solvent. J Comp Chem 34:1890-1898. https://doi.org/10.1002/jcc.23334

9. Stengle TR, Hosseini SM, Basiri HG, Williamson KL (1984) NMR chemical shifts of xenon in aqueous solutions of amphiphiles: A new probe of the hydrophobic environment. J Solution Chem 13:779-787. https://doi.org/10.1007/BF00647693

10. Aprile E, Doke T (2010) Liquid xenon detectors for particle physics and astrophysics. Rev Mod Phys 82:20532097. https://doi.org/10.1103/RevModPhys.82.2053

11. Jameson CJ, Sears DN, de Dios AC (2003) The ${ }^{129} \mathrm{Xe}$ nuclear shielding tensor surfaces for Xe interacting with rare gas atoms. J Chem Phys 118:2575. https://doi.org/10.1063/1.1534093.

12. Jankowska M, Kupka T, Stobiński L, Faber R, Lacerda Jr. EG, Sauer SPA (2016) Spin-orbit ZORA and four-component Dirac-Coulomb estimation of relativistic corrections to isotropic nuclear shieldings and chemical shifts of noble gas dimers. J Comput Chem 37:395-403. https://doi.org/10.1002/jcc.24228.
13. Hess B, Kutzner C, van der Spoel D, Lindahl E (2008) GROMACS 4: Algorithms for Highly Efficient, Load-Balanced, and Scalable Molecular Simulation. J Chem Theory Comput 4:435-447. https://doi.org/10.1021/ct700301q

14. Van Der Spoel D, Lindahl E, Hess B, Groenhof G, Mark AE, Berendsen HJC (2005) GROMACS: fast, flexible, and free. J Comput Chem 26:1701-1718. https://doi.org/10.1002/jcc.20291.

15. Verlet L, Weis J-J (1972) Perturbation theory for the thermodynamic properties of simple liquids. Mol Phys 24:1013-1024. https://doi.org/10.1080/00268977200102111.

16. Berendsen HJC, Postma JPM, van Gunsteren WF, DiNola A, Haak JR (1984) Molecular dynamics with coupling to an external bath. J Chem Phys, 81:3684:3690, https://doi.org/10.1063/1.448118

17. Bussi G, Donadio D, Parrinello M (2007) Canonical sampling through velocity rescaling. J Chem Phys, 126:014101, https://doi.org/10.1063/1.2408420

18. Frisch MJ, Trucks GW, Schlegel HB, Scuseria GE, Robb Ma, Cheeseman JR, Scalmani G, Barone V, Mennucci B, Petersson Ga, Nakatsuji H, Caricato M, Li X, Hratchian HP, Izmaylov aF, Bloino J, Zheng G, Sonnenberg JL, Hada M, Ehara M, Toyota K, Fukuda R, Hasegawa J, Ishida M, Nakajima T, Honda Y, Kitao O, Nakai $\mathrm{H}$, Vreven T, Montgomery Ja, Peralta JE, Ogliaro F, Bearpark M, Heyd JJ, Brothers E, Kudin KN, Staroverov VN, Kobayashi R, Normand J, Raghavachari K, Rendell a, Burant JC, Iyengar SS, Tomasi J, Cossi M, Rega N, Millam JM, Klene M, Knox JE, Cross JB, Bakken V, Adamo C, Jaramillo J, Gomperts R, Stratmann RE, Yazyev O, Austin aJ, Cammi R, Pomelli C, Ochterski JW, Martin RL, Morokuma K, Zakrzewski VG, Voth Ga, Salvador P, Dannenberg JJ, Dapprich S, Daniels aD, Farkas Ö, Foresman JB, Ortiz JV, Cioslowski J, Fox DJ, Montgomery Jr Ja Gaussian 09, Rev A.1 (2009)

19. de Oliveira PJP, Barros CL, Jorge FE, Canal Neto A, Campos M (2010) Augmented Gaussian basis set of double zeta valence quality for the atoms $\mathrm{Rb}$ and $\mathrm{Y}$ Xe: Application in DFT calculations of molecular electric properties. J Mol Struct THEOCHEM 948:43-46. https://doi.org/10.1016/j.theochem.2010.02.017

20. Campos CT, Jorge FE (2013) Triple zeta quality basis sets for atoms $\mathrm{Rb}$ through $\mathrm{Xe:}$ application in $\operatorname{CCSD}(\mathrm{T})$ atomic and molecular property calculations. Mol Phys 111:167-173. https://doi.org/10.1080/00268976.2012.709282.

21. Martins LSC, de Souza FAL, Ceolin GA, Jorge FE, de Berrêdo RC, Campos CT (2013) Augmented Gaussian basis sets for the elements $\mathrm{K}, \mathrm{Sc}-\mathrm{Kr}, \mathrm{Rb}$, and $\mathrm{Y}-\mathrm{Xe}$ : Application in HF, MP2, and DFT calculations of molecular electric properties. Comput Theor Chem 1013:6269. https://doi.org/10.1016/j.comptc.2013.03.004.

22. Schuchardt KL, Didier BT, Elsethagen T, Sun L, Gurumoorthi V, Chase J, Li J, Windus TL (2007) Basis Set Exchange: A Community Database for Computational Sciences. J Chem Inf Model 47:1045-1052. https://doi.org/10.1021/ci600510j.

23. Feller D (1996) The role of databases in support of computational chemistry calculations. J Comput Chem 17:1571-1586. https://doi.org/10.1002/"(SICI)1096987X(199610)17:13¡1571::AID-JCC9 ¿3.0.CO;2-P”.

24. Saue T, Visscher L, Jensen HJAa, Bast R with contributions from Bakken V, Dyall KG, Dubillard S, Ekström U, Eliav E, Enevoldsen T, Faßhauer E, Fleig T, Fossgaard 
O, Gomes ASP, Helgaker T, Lærdahl JK, Lee YS, Henriksson J, Iliaš M, Jacob CR, Knecht S, Komorovský S, Kllie O, Larsen CV, Nataraj HS, Norman P, Olejniczak G, Olsen J, Park YC, Pedersen JK, Pernpointner M, di Remigio R, Ruud K, Sałek P, Schimmelpfennig B, Sikkema J, Thorvaldsen AJ, Thyssen J, van Stralen J, Villaume S, Visser O, Winther T, Yamamoto S DIRAC, a relativistic ab initio electronic structure program, Release DIRAC14 (2014) (see www.diracprogram.org)

25. Saue T, Fægri Jr K, Helgaker T, Gropen O (1997) Principles of direct 4-component relativistic SCF: Applications to cesium auride. Mol Phys 91:937-950. https://doi.org/10.1080/002689797171058

26. Aucar G, Saue T, Visscher, L, Jensen HJAa (1999) On the origin and contribution of the diamagnetic term in four-component relativistic calculations of magnetic properties. J Chem Phys 110:6208-6218. https://doi.org/10.1063/1.479181

27. Visscher L, Enevoldsen T, Saue T, Jensen HJAa, Oddershede J (1999) Relativistic calculations of the NMR shielding and indirect spin-spin coupling tensors in the hydrogen halides. J Comp Chem 20:1262-1273. https://doi.org/10.1002/(SICI)1096987X(199909)20:12;1262::AID-JCC6 ¿3.0.CO;2-H

28. Dyall KG (2006) Relativistic quadruple-zeta and revised triple-zeta and double-zeta basis sets for the $4 \mathrm{p}$, 5p, and 6p elements. Theor Chem Acc 115:441-447. https://doi.org/10.1007/s00214-006-0126-0 


\section{Support Information: Theoretical Study of the NMR Chemical Shift of Xe in Supercritical Condition}

Evanildo G. Lacerda Jr. Stephan P. A. Sauer Kurt V. Mikkelsen Kaline Coutinho Sylvio Canuto

January 21, 2018

\section{B3LYP results for Xe dimers}

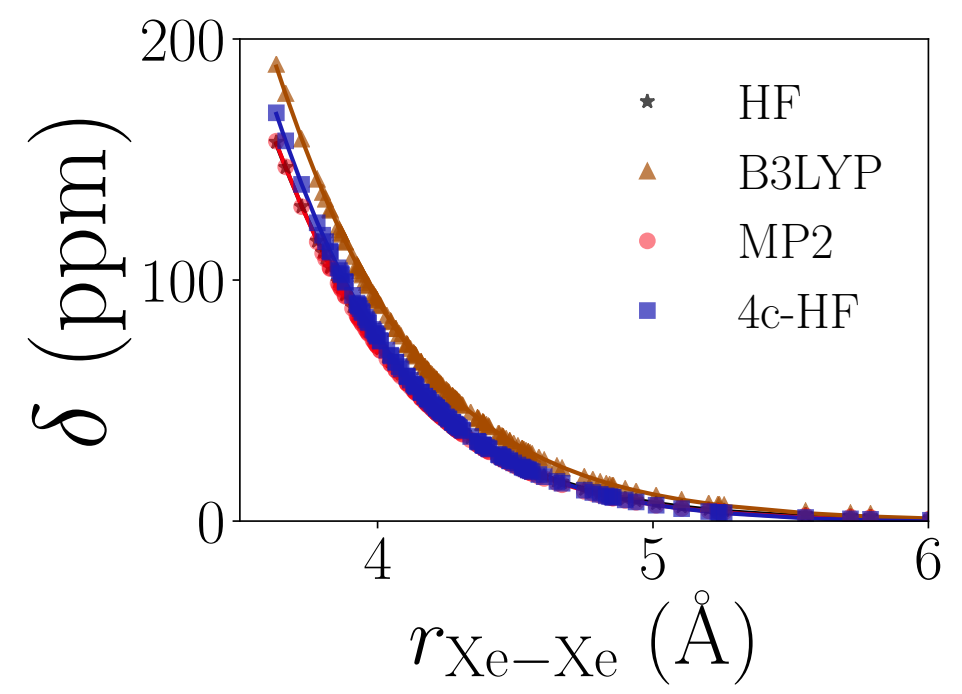

Figure 1: Calculated chemical shifts of a Xe dimer in ppm as a function of the interatomic distance. 


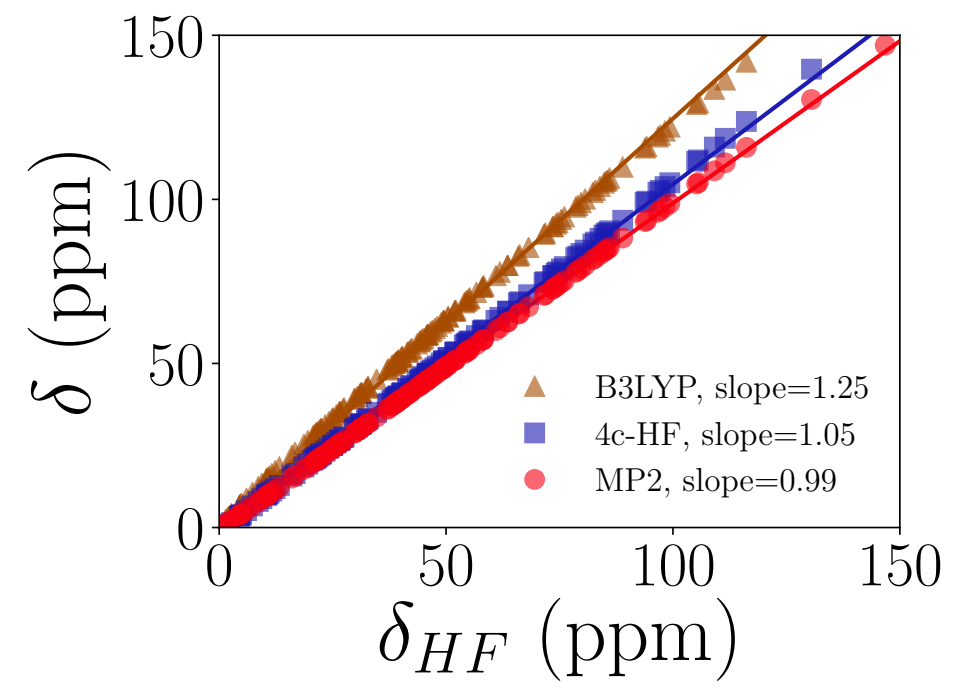

Figure 2: The correlation of the chemical shifts in Xe dimers calculated at B3LYP, MP2 and 4c-HF levels with the corresponding HF values in ppm. 